\title{
Oligodendrocyte Progenitor Cell Susceptibility to Injury in Multiple Sclerosis
}

\author{
Qiao-Ling Cui, ${ }^{* \dagger}$ Tanja Kuhlmann, ${ }^{\ddagger}$ Veronique E. Miron, ${ }^{*}$ Soo Yuen Leong, ${ }^{*}$ Jun Fang, ${ }^{\dagger}$ Pavel Gris, ${ }^{*}$ Timothy E. Kennedy, ${ }^{*}$
} Guillermina Almazan, ${ }^{\dagger}$ and Jack Antel ${ }^{*}$

From the Montreal Neurological Institute* and the Department of Pharmacology and Therapeutics, ${ }^{\dagger}$ McGill University, Montreal, Quebec, Canada; and the Institute of Neuropathology, ${ }^{\ddagger}$ University Hospital Münster, Münster, Germany

Accepted for publication April 8, 2013.

Address correspondence to Jack Antel, M.D., Neuroimmunology Unit, Montreal Neurological Institute, McGill University, 3801 University St, Montreal, QC H3A 2B4, Canada. E-mail: jack.antel@mcgill.ca.

\begin{abstract}
Remyelination in multiple sclerosis (MS) is often incomplete. In experimental models, oligodendrocyte progenitor cells (OPCS) rather than previously myelinating oligodendrocytes (OLs) are responsible for remyelination. This study compares the relative susceptibility of adult human OPCs and mature OLs to injury in actively demyelinating MS lesions and under in vitro stress conditions. In all lesions $(n=20)$, the number of OLs (Olig2 weak/NogoA positive) was reduced compared to control white matter (mean $38 \pm 4 \%$ of control value). In 11 cases, OPC numbers (0lig2 strong; NogoA negative) were also decreased; in eight of these, the reduction was greater for OPCs than for OLs. In the other nine samples, OPC numbers were greater than control white matter, indicating ongoing OPC migration and/or proliferation. Analysis of co-cultures with rat dorsal root ganglia neurons confirmed that OPCs were more capable of contacting and ensheathing axons than OLs. In isolated culture under stress conditions (withdrawal of serum/ glucose and/or antioxidants), OPCs showed increased cell death and reduced process extension compared to OLs. Under all culture conditions, OPCs up-regulated expression of genes in the extrinsic proapoptotic pathway, and had increased susceptibility to tumor necrosis factor-induced cell death as compared to OLs. Our data suggest that susceptibility of OPCs to injury within the MS lesion environment contributes to the limited remyelination in MS. (Am J Pathol 2013, 183: 516-525; http://dx.doi.org/10.1016/ j.ajpath.2013.04.016)
\end{abstract}

The extent of remyelination in multiple sclerosis (MS) lesions is variable and often incomplete. The source of cells mediating the remyelination and the basis for their limited effectiveness remains to be fully defined. Recent animal experiments using genetic fate mapping techniques ${ }^{1,2}$ implicate oligodendrocyte progenitor cells (OPCs), and not mature previously myelinating oligodendrocytes (OLs), as the cells responsible for remyelination. $^{3-5}$ OPCs can be identified within active MS lesions in humans, but their number and capacity to differentiate decreases with disease duration. ${ }^{6}$ Our study pursues the postulate that injury/loss of OPCs during the acute lesion phase of MS can be a contributing factor for suboptimal remyelination.

OL/myelin injury associated with inflammation is the pathological hallmark of an early active MS lesion. The precise mechanisms underlying such injury remain to be defined. Implicated mechanisms, which could be acting concurrently, include direct immune-mediated injury of neural targets such as by cell- or antibody-mediated cytotoxicity and/or inflammationassociated molecules, including tumor necrosis factor$\alpha$ (TNF- $\alpha){ }^{7}$ There is increasing recognition that injury can also arise from changes in the microenvironment that induce metabolic cellular insults. ${ }^{8-11}$ In some MS lesions, there is pronounced OL loss and dying back of the terminal processes of the remaining cells ${ }^{12-14}$; this oligodendrogliopathy resembles findings observed distal to an ischemic insult. ${ }^{8,9}$ Mitochondrial failure has also been recognized in OLs, as well as in neurons/ axons, in MS lesions. ${ }^{15-17}$

The extent to which OPCs may be susceptible to similar injury as mature OLs, with which they share a common microenvironment, in MS lesions remains to be defined. Rodent-based in vitro studies have reported enhanced susceptibility of cultured OPCs to effecter cytokines ${ }^{18}$ and

Supported by operating grants from the Multiple Sclerosis Society of Canada (G.A. and J.A). 
the excitotoxin glutamate compared to more mature OLs. ${ }^{19-21}$ Ziabreva et al, ${ }^{22}$ however, found that inhibitors of mitochondrial respiratory complex IV induced greater injury of OLs matured in vitro from the neonatal rat brain compared to OPCs.

In the current study, we show that relative numbers of OPCs can be more reduced than mature OLs in actively demyelinating MS lesions. Using cells isolated from noninflamed adult human brain samples, we provide evidence that OPCs (A2B5 antibody selected) rather than previously myelinating mature OLs have greater capacity to ensheath axons. Furthermore, we demonstrate that cultured OPCs have enhanced vulnerability to injury compared to mature OLs when placed under metabolic stress conditions (withdrawal of serum/glucose and/or antioxidants); under all culture conditions, OPCs had higher expression of genes in the extrinsic proapoptotic signaling cascade and were more susceptible to subsequent TNF- $\alpha$-induced injury.

\section{Materials and Methods}

\section{Analysis of OPCs and OLs in Early MS Lesion Stages}

We analyzed surgically obtained brain samples containing actively demyelinating lesions from MS patients for the expression of OL lineage transcription factor 2 (Olig2) as a marker of OPCs and a member of a family of integral membrane proteins termed reticulons (NogoA) as a marker of mature OLs. ${ }^{6}$ All actively demyelinating lesions (active) showed macrophage infiltration and/or microglial activation and presence of myelin degradation products within macrophages $\left[2^{\prime}, 3^{\prime}\right.$-cyclic nucleotide $3^{\prime}$-phosphodiesterse (CNP), myelin OL glycoprotein (MOG), myelin associated glycoprotein (MAG)]..$^{23,24}$ Two sets of samples were evaluated, namely, samples included in an earlier study $(n=16)^{6}$ and four new samples. Each was compared to brain tissue specimens obtained from non-MS cases and immunostained at the same time; the surgically derived MS samples did not consistently contain normal-appearing white matter. OPCs are defined as being Olig2 strong and NogoA negative (Olig2 ${ }^{\text {str }}$ ); mature OLs are Olig2 weak/NogoA strong $\left(\mathrm{NogoA}^{\mathrm{str}}\right)$. Immunohistochemistry was performed as described previously. ${ }^{6}$ The study was approved by the ethics committees of the University of Göttingen, University Hospital of Münster, and McGill University.

\section{Isolation of Adult Human OPCs and OLs}

Brain tissue was obtained from adults undergoing surgical resections as treatment for non-tumor-related intractable epilepsy in accordance with the guidelines set by the Biomedical Ethics Unit of McGill University. The material came predominantly from temporal lobe white matter and did not include subependymal regions. Tissue specimens were enzymatically digested and placed on a linear 30\% Percoll density gradient (Pharmacia Biotech, Piscataway, NJ).
Microglia were separated and removed by an initial adhesion step in which the total cell fraction was cultured for 24 hours in noncoated flasks. In the floating cell fraction, $40 \%$ to $50 \%$ of the cells reacted with R-monoclonal antibody, which recognizes mainly galactocerebroside $(\mathrm{GalC})^{25}$; whereas $<5 \%$ of the cells were microglia and astrocytes. ${ }^{26}$ This cellular fraction was subjected to immunomagnetic bead selection with the A2B5 antibody (IgM), which recognizes gangliosides. ${ }^{27}$ Cell recovery in the positively selected fraction (referred to as $\mathrm{A} 2 \mathrm{~B}^{+}{ }^{+}$cells, or OPCs) was approximately $3 \%$ to $8 \%$ of initial numbers; approximately $20 \%$ of the cells were $\mathrm{GalC}^{+}$as determined by flow cytometry. For the nonselected fraction (referred to as the $\mathrm{A} 2 \mathrm{~B} 5{ }^{-}$fraction, or OLs), $50 \%$ to $60 \%$ of cells expressed GalC (data not shown). After 7 days in dissociated culture, $20 \%$ to $25 \%$ of cells in the $\mathrm{A} 2 \mathrm{~B}^{+}{ }^{+}$fraction were reactive with $\mathrm{O} 1$ antibody (recognizes $\mathrm{GalC}^{28}$ ), whereas almost all (85\% to 95\%) cells in the negatively selected fraction were $\mathrm{O}^{+}$(Supplemental Figure S1).

An Affymetrix-based microarray comparison (Affymetrix, Santa Clara, CA) between the two cell fractions at the time of isolation indicated that the $\mathrm{A}_{2} \mathrm{~B}^{+}$cells had increased expression of mRNA for platelet-derived growth factor receptor (PDGFR) $\alpha$ (mean fold increase for the 36 probes of the PDGFR $\alpha$ gene $=2.14 ; P=0.6 \times 10^{-9}$, paired $t$-test) and for protein tyrosine phosphatase, receptortype, Z1 (PTPRZ1) (mean fold increase $=6.64 ; P=0.19 \times$ $10^{-9}$ ) (data not shown), markers of OPC, ${ }^{29}$ supporting that this fraction is enriched in progenitor cells.

\section{Human OPC or OL and Rat Dorsal Root Ganglion Neuron Co-Cultures}

Purified dorsal root ganglion neuron (DRGN) cultures were prepared from Sprague-Dawley rat embryos as described previously. ${ }^{30}$ The cells were maintained with $12.5 \mathrm{ng} / \mathrm{mL}$ nerve growth factors (NGF) in serum-free N1-supplemented medium for 3 weeks before the addition of human cells to allow differentiation of neurons and extension of a network of neurites competent for myelination. OPCs $\left(\mathrm{A}^{2} \mathrm{~B}^{+}\right)$or $\mathrm{OLs}$ $\left(\mathrm{A} 2 \mathrm{~B} 5^{-}\right)$at a density of $0.7 \times 10^{5}$ cells $/ \mathrm{cm}^{2}$ suspended in defined medium were added to DRGN cultures. Defined medium consisted of Dulbecco's modified essential medium-F12 supplemented with N1 (Sigma-Aldrich, Oakville, ON, Canada), $0.01 \%$ bovine serum albumin, $1 \%$ penicillinstreptomycin, and B27 supplement (Invitrogen, Burlington, $\mathrm{ON}$, Canada), $10 \mathrm{ng} / \mathrm{mL}$ platelet-derived growth factor (PDGF)-AA, $10 \mathrm{ng} / \mathrm{mL}$ basic fibroblast factor (bFGF), and 2 $\mathrm{nmol} / \mathrm{L}$ triiodothyronine (T3) (Sigma-Aldrich) (referred to as $\mathrm{DM}+\mathrm{GF}$ ). The OPCs and OLs were used immediately after fractionation. The extent of cell survival, differentiation, and axonal ensheathment was analyzed after 5 weeks of co-culture.

\section{Isolated OPC and OL Cell Cultures}

To assess the relative susceptibility of OPCs and OLs to injury (cell loss, TUNEL-labeled nuclei, and process retraction) 
Table 1 Primary and Secondary Antibodies Used in This Study

\begin{tabular}{|c|c|c|c|c|c|}
\hline Antibody & Type & Species & Clonality & Dilution & Source \\
\hline Olig2 & IgG & Rabbit & Polyclonal & $1: 200$ & Immuno-Biological Laboratories (Spring Lake Park, MN) \\
\hline 04 & $\operatorname{Ig} M$ & Hybridoma & Monoclonal & $1: 50$ & Sommer et $\mathrm{al}^{28}$ \\
\hline GalC & $\operatorname{IgG3}$ & Hybridoma & Monoclonal & $1: 50$ & Ranscht et $\mathrm{al}^{25}$ \\
\hline 01 & $\operatorname{Ig} M$ & Hybridoma & Monoclonal & $1: 25$ & Sommer et $\mathrm{al}^{28}$ \\
\hline NogoA & IgG1 & Mouse & Monoclonal & $1: 1000$ & Gift from Dr. Martin Schwab \\
\hline MBP & $\mathrm{IgG}_{2 \mathrm{~b}}$ & Mouse & Monoclonal & $1: 500$ & Sternberger (Lutherville, MD) \\
\hline Caspr & $\operatorname{IgG}$ & Rabbit & Polyclonal & $1: 1000$ & Gift from Dr. David Colman \\
\hline Sodium channel & $\operatorname{IgG1}$ & Mouse & Monoclonal & $1: 1000$ & Sigma-Aldrich \\
\hline N52 & $\mathrm{IgG}_{1}$ & Mouse & Monoclonal & $1: 1000$ & Sigma-Aldrich \\
\hline A2B5-microbead & $\operatorname{Ig} M$ & Rat anti-mouse & Monoclonal & $1: 4$ & Miltenyi Biotech (Auburn, CA) \\
\hline IgM-FITC or TxR & & Goat anti-mouse & Monoclonal & $1: 100$ & Jackson ImmunoResearch (Westgrove, PA) \\
\hline $\mathrm{IgG}_{2 \mathrm{~b}}-\mathrm{FITC}$ or $\mathrm{TxR}$ & & Goat anti-mouse & Monoclonal & $1: 100$ & Biosource (Camarillo, CA) \\
\hline $\mathrm{IgG}_{1}-\mathrm{FITC}$ or $\mathrm{TxR}$ & & Goat anti-mouse & Monoclonal & $1: 100$ & Molecular Probes (Eugene, OR) \\
\hline $\mathrm{Cy} 3$ & & Goat anti-rabbit & Polyclonal & $1: 100$ & Jackson ImmunoResearch \\
\hline
\end{tabular}

under metabolically stressful conditions, OPCs or OLs were plated $\left(2.5 \times 10^{5}\right.$ cells per $\left.\mathrm{mL}\right)$ on polylysine-coated chamber slides and cultured for 4 to 10 days under conditions that ranged from optimal to metabolically deprived; these involved defined media supplemented with growth factors (DM+GF); minimum essential medium (MEM) with $5 \%$ fetal calf serum (FCS) (MEM+FCS), DM+GF without antioxidants (DM+GF-AO), and MEM depleted of glucose and serum (MEM alone), respectively. In additional experiments, 100 $\mathrm{ng} / \mathrm{mL} \mathrm{TNF}-\alpha$ was added to both cell types for 48 hours under the culture conditions indicated in the Results section.

\section{Immunocytochemistry}

For assessment of cell-surface markers, cells in either isolated or co-cultures were incubated with primary antibodies $(\mathrm{O} 4$ and O1) (Table 1) for 30 minutes at $4{ }^{\circ} \mathrm{C}$ then fixed in $4 \%$ paraformaldehyde for 10 minutes at $4^{\circ} \mathrm{C}$, followed by blocking with HHG (1 mmol/L HEPES, $2 \%$ horse serum, $10 \%$ goat serum, Hanks' balanced salt solution) for 10 minutes. Cultures were incubated with secondary antibodies conjugated with fluorescent dyes (Table 1) for 30 minutes at $4^{\circ} \mathrm{C}$. Antibody isotype controls showed low nonspecific staining (data not shown). Cell nuclei were stained with 10 $\mu \mathrm{g} / \mathrm{mL}$ Hoechst 33258 (Invitrogen) for 10 minutes at room temperature. For isolated cultures, $\mathrm{TUNEL}^{+}$cells were identified with a commercial kit (Promega, Madison, WI). Relative process area per cell was calculated as a percentage of that measured in the $\mathrm{DM}+\mathrm{GF}$ condition using ImageJ software version 1.47f (NIH, Bethesda, MD). For co-culture studies, slides were visualized by confocal microscopy on a Zeiss LSM 510 (Zeiss, Oberkochen, Germany) using a $63 \times$ oil objective and analyzed with the LSM 510 Image browser software version 4.2.0.121 (Zeiss). The optical thickness was $<1.0 \mu \mathrm{m}$ for each channel. For isolated cell cultures studies, slides were mounted using gel/mount (Biomeda Corporation, Foster City, CA), and signals visualized by epifluorescent microscopy (Leica, Montreal, QC, Canada) and OpenLab imaging software version 5.5.2 (OpenLab, Florence, Italy).

\section{$\mathrm{RT}^{2}$ Profiler Apoptosis PCR Array}

The expression of 84 apoptosis-related genes was examined using the 84-gene human apoptosis PCR array kit (PAHS012; SABiosciences/Qiagen, Mississauga, ON, Canada). Specific genes included are detailed in Table 2. RNA was extracted from the $\mathrm{A} 2 \mathrm{~B}^{+}$selected and $\mathrm{A} 2 \mathrm{~B} 5^{-}$oligodendroglial lineage cells immediately after fractionation (day 0 ) or after 6 days in culture. Total RNA was reverse-transcribed into cDNA using the $\mathrm{RT}^{2}$ First Strand Kit (SABiosciences), mixed with $\mathrm{RT}^{2}$ qPCR Master Mix containing SYBR Green (SABiosciences), and aliquoted in equal volumes to each well of the real-time PCR arrays. The threshold cycle $(\mathrm{Ct})$ of each gene was determined and normalized using multiple housekeeping genes and subsequently analyzed by $\mathrm{RT}^{2}$ Profiler PCR Array Data Analysis software version 3.5. Data from three separate cell preparations were analyzed.

\section{Data Analysis}

Results are presented either as means \pm SEM or as fold/ percent difference over control for corresponding time points. The number of individual experiments for each study is indicated in the Results section. Statistical significance was determined by two-way analysis of variance, followed by either Dunnett's or Bonferroni's multiple comparison tests. Probability values $<0.05$ were considered statistically significant.

\section{Results}

Evaluation of OPC and Mature OL Numbers in MS Lesions in Situ

In an earlier publication, ${ }^{6}$ we reported the presence of OPCs in most early and chronic MS lesions with a significant increase of OPCs in the periplaque white matter (PPWM). Now, we assess the relative loss of OPC as compared to mature OLs in individual actively demyelinating lesions; the 
Table 2 Categorization of PCR Array Genes

\begin{tabular}{|c|c|c|c|}
\hline \multicolumn{3}{|l|}{ Proapoptotic } & \multirow[b]{2}{*}{ Antiapoptotic } \\
\hline Extrinsic & Intrinsic & Effector & \\
\hline$\overline{C D 27^{*}}$ & $A B L 1$ & CASP1 & AKT1 \\
\hline$C D 40^{*}$ & APAF1 & CASP14 & $B A G 1$ \\
\hline CD $40 L G$ & $B A D$ & CASP2 & $B A G 3$ \\
\hline CD70 & $B A K 1$ & CASP3 & $B A G 4$ \\
\hline CRADD & $B A X$ & CASP4 & $B C L 2$ \\
\hline DAPK1 & $B C L 10$ & CASP5 & $B C L 2 A 1$ \\
\hline$F A D D$ & $B C L 2 L 10$ & CASP6 & $B C L 2 L 1$ \\
\hline$F A S^{*}$ & $B C L 2 L 11$ & CASP7 & $B C L 2 L 2$ \\
\hline FASLG & $B C L A F 1$ & CASPg & BFAR \\
\hline$L T A$ & $B I D$ & & NAIP \\
\hline$\angle T B R^{*}$ & BIK & & $B I R C 2$ \\
\hline$T N F$ & BNIP1 & & $B I R C 3$ \\
\hline TNFRSF10A* & BNIP2 & & $X I A P$ \\
\hline TNFRSF1OB* & BNIP3 & & BIRC6 \\
\hline TNFRSF1A* & $B N I P 3 L$ & & BIRC8 \\
\hline TNFRSF21 * & CARD6 & & $B R A F$ \\
\hline TNFRSF25* & CARD8 & & $I G F 1 R$ \\
\hline TNFRSF9* & CFLAR & & MCL1 \\
\hline TNFSF10 & CIDEA & & TRAF2 \\
\hline TNFSF8 & CIDEB & & TRAF3 \\
\hline TRADD & $D F F A$ & & TRAF4 \\
\hline CASP10 & GADD45A & & TNFRSF11B* \\
\hline \multirow[t]{8}{*}{ CASP8 } & HRK & & \\
\hline & NOD1 & & \\
\hline & NOL3 & & \\
\hline & PYCARD & & \\
\hline & RIPK2 & & \\
\hline & TP53 & & \\
\hline & ТР53ВP2 & & \\
\hline & TP73 & & \\
\hline
\end{tabular}

*Member of TNF receptor superfamily.

results are expressed as a percentage of mean values for the corresponding cell type found in nondemyelinating control white matter sections stained at the same time. As shown in Figure 1, A and B, the number of mature OLs $\left(\operatorname{NogoA}^{\mathrm{str}}\right)$ was reduced in all 20 active lesions examined compared to control white matter (mean $38 \pm 4 \%$ of control value). The relative number of OPCs $\left(\mathrm{Olig} 2^{\text {str }}\right)$ in actively demyelinating lesions was reduced in 11 of 20 cases compared to control sections (Figure 1A). In eight of these, the relative percentage reduction in OPCs was greater than in mature OLs. In the other nine cases (Figure 1B), the relative number of OPCs was increased compared to control white matter sections. The mean decrease in the percentage of mature OLs in the cases where OPCs are reduced was not significantly different when compared to the cases with increased OPC numbers (36.8 \pm $5.5 \%$ versus $44.2 \pm 3.5 \% ; P=0.27$ ).

In a subset of these tissue samples $(n=11)$, PPWM was also available; OPC numbers were lower in the active lesion areas compared to PPWM in the corresponding tissue sample (Figure 1C). In four cases, PPWM OPC numbers were higher than in control tissue, whereas OPC numbers were reduced in the lesion (Figure 1C). In four other cases, OPCs numbers were reduced in both lesions and PPWM, with reduction being greater in the active lesions (Figure 1C). In the remaining three cases, OPC numbers were relatively increased in both lesions and PPWM compared to control tissue (Figure 1C). The relative number of mature OLs was reduced in the PPWM compared to control white matter (mean $55 \pm 5 \%$ of control value). Examples of immunostaining for OPCs $\left(\mathrm{Olig}_{2}{ }^{\mathrm{str}}\right)$ and mature OLs $\left(\mathrm{NogoA}^{\mathrm{str}}\right)$ in active lesions and PPWM are illustrated in Figure 1D.

\section{Comparison of Axonal Ensheathing Capacity of A2B5 ${ }^{+}$ OPCs and Mature OLs Co-Cultured with DRGNs}

Figure 2, A and B, illustrates the extent of cell survival, differentiation, and axonal ensheathment of OPCs and OLs after 5 weeks in co-culture with DRGNs. OPCs establish contacts with axons and begin the ensheathment process (Figure 2A). Clustering of the paranodal protein contactinassociated protein (Caspr) indicates the initial formation of

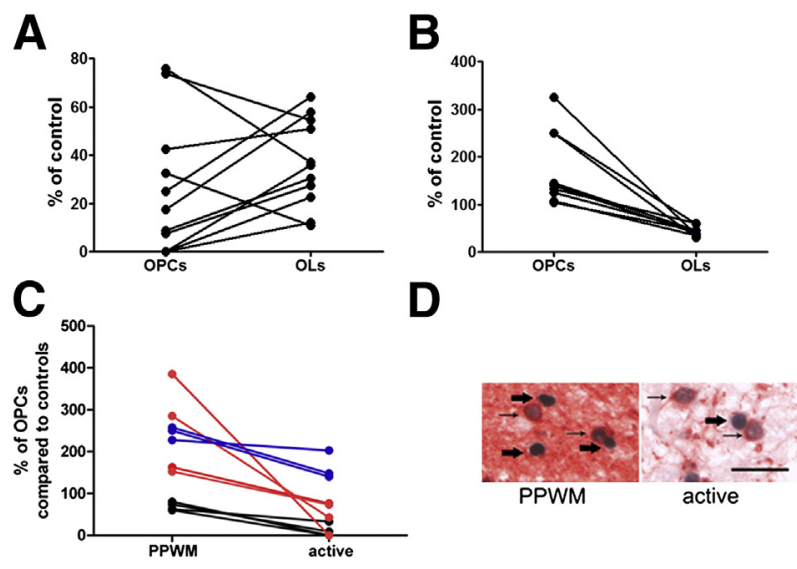

Figure 1 Comparison of $\mathrm{OPC}$ and mature $\mathrm{OL}$ numbers in MS active lesions in situ. MS brain section samples $(n=20)$ containing demyelinating active lesions were analyzed; of these, 11 contained PPWM, which was also analyzed. We quantified the number of OPC (Olig2 $\left.{ }^{\text {str }}\right)$ and mature OLs $\left(\mathrm{NogoA}^{\mathrm{str}}\right)$ using a standardized ocular grid. For 11 cases with OPC values $<$ control white matter $(\mathbf{A})$ and 9 cases with $\mathrm{OPC}$ values $>$ control white matter (B), the relative number of OPCs and corresponding mature OLs in actively demyelinating lesions for individual cases is expressed as a percentage of corresponding cells in normal control white matter. C: Comparison between the percentage of OPCs relative to normal control white matter in active demyelinating lesions with percentage of OPCs relative to normal control white matter in corresponding PPWM for individual cases: red lines indicate four cases where OPC numbers in PPWM were higher than in control tissue values, whereas OPC numbers were reduced in actively demyelinating lesions compared to control tissue values; black lines indicate four cases in which OPCs numbers was reduced in both lesions and PPWM; blue lines indicate three other cases where OPC numbers were relatively increased in both lesions and PPWM compared to control tissue. The mean number of OPCs in the control tissue sections was 40 cells $/ \mathrm{mm}^{2}$ (range, 39 to 44) for the first set of sections and 79 cells $/ \mathrm{mm}^{2}$ (range, 70 to 90 ) for the second set of sections. The mean number of OLs in the control tissue sections was 817 cells $/ \mathrm{mm}^{2}$ (range, 577 to 1388) for the first set of sections and 1240 cells $/ \mathrm{mm}^{2}$ (range, 988 to 1500) for the second set of sections. D: Immunostained brain section containing PPWM and the active lesion. OPCs were identified by strong expression of Olig2 but lack of NogoA (Olig2 $2^{\text {str }}$, thick arrows), whereas mature OLs expressed NogoA and low levels of Olig2 ( $\operatorname{Nogo}^{\text {str }}$, thin arrows). Scale bar $=25 \mu \mathrm{m}$. 
axonal domains; however, sodium channel clustering (Figure 2, $\mathrm{C}, \mathrm{D}, \mathrm{G}$, and $\mathrm{H}$ ), which is fundamental to the nodes of Ranvier formation, is not evident. This pattern indicates that the process of myelination has begun but remains incomplete. By contrast, for the mature OLs, there was limited process formation, axonal contact, or ensheathment (Figure 2B). The few sites of Caspr aggregation occurred between axons and OL cell bodies rather than between cellular processes (Figure 2, E, F, I, and J), in contrast to OPC/axon interactions. Differences in axon ensheathment by OPCs and OLs and in Caspr redistribution along axons are quantified in Figure 2, $\mathrm{K}$ and $\mathrm{L}$.

\section{Comparison of OPC and Mature OL Responses to Metabolic Injury in Vitro}

\section{Comparison of Injury Response of $\mathrm{A}_{2} \mathrm{~B}^{+} \mathrm{OPCs}$ and $\mathrm{A} 2 \mathrm{~B} 5^{-}$}

\section{OLs in Isolated Cell Cultures}

The relative survival and process outgrowth of OPCs or OLs after 6 days in isolated cell culture under different conditions are quantified in Figure 3, A-C. Figure 3, D-K, illustrates these findings. Under optimal conditions $(\mathrm{DM}+\mathrm{GF})$, both OPCs and OLs survive well $\left(<5 \% \mathrm{TUNEL}^{+}\right.$cells $)$and extend cellular processes. Relative process area per cell (Figure 3C) is greater for OPCs $\left(10.5 \pm 0.4 \mu \mathrm{mol} / \mathrm{L}^{2} /\right.$ cell $)$ compared to OLs $\left(8.6 \pm 0.3 \mu \mathrm{mol} / \mathrm{L}^{2} /\right.$ cell; $P<0.05, n=4$ separate cultures). Under the most metabolically deficient conditions, MEM without glucose and serum (MEM alone), there was a significant increase in the percentage of TUNELpositive OPCs as compared to OLs $(25 \pm 2.7 \%$ versus $15 \pm$ $1.2 \%, P<0.001$ ) (Figure 3A) at day 6. The total number of OPCs decreased from $212 \pm 2$ per field under optimal conditions $(\mathrm{DM}+\mathrm{GF})$ to $95 \pm 5$ in MEM alone, accounting for a $56 \%$ reduction (Figure 3B). For mature OLs, the mean number of surviving cells in MEM was $142 \pm 8$ compared to $176 \pm 6$ in $\mathrm{DF}+\mathrm{GM}$, accounting for only $19 \%$ reduction. The relative process area per cell in MEM was significantly reduced for both cell types ( $71 \pm 1.5 \%$ for OPCs versus $60 \pm$ $2 \%$ for OLs) as compared to DM+GF (Figure 3C). The relative reduction in OPC process area was significantly greater than for OLs $(P<0.01)$.

Under the less severe conditions of MEM+FCS, neither OPCs nor OLs showed enhanced cell death as measured by the percentage of $\mathrm{TUNEL}^{+}$cells or the total number of surviving cells compared to optimal conditions at day 6 (Figure 3, A and B). However, by 3 weeks, the numbers of surviving OPCs decreased by $56 \pm 1.7 \%$, whereas surviving OLs decreased by $34 \pm 3.5 \%(P<0.01)$ (data not shown). At day 6 , the relative process area per cell was significantly reduced for both cell types $(45 \pm 2.1 \%$ and $38 \pm 1.8 \%$ of values compared to that observed under optimal conditions) (Figure 3C). Under culture conditions in which antioxidants were removed from DM+GF (DM+GF-AO), OPCs, but not the OLs, showed an increase in the percentage of $\mathrm{TUNEL}^{+}$cells (Figure 3A). The relative process area per cell for both OPCs and OLs was reduced as compared to $\mathrm{DM}+\mathrm{GF}$ (Figure 3C).
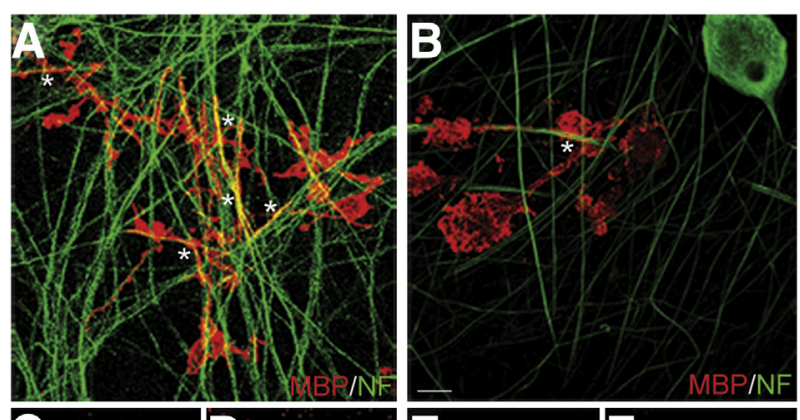
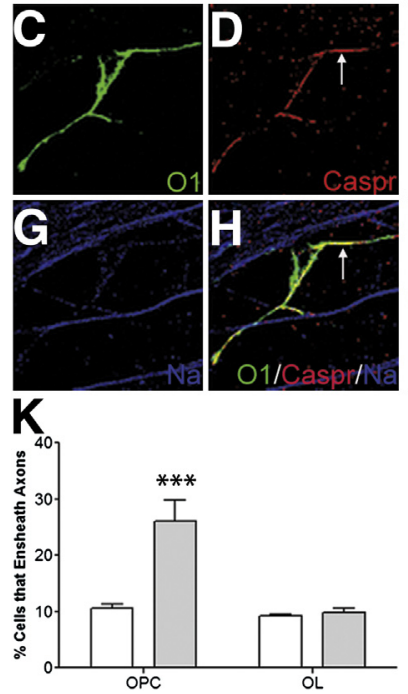
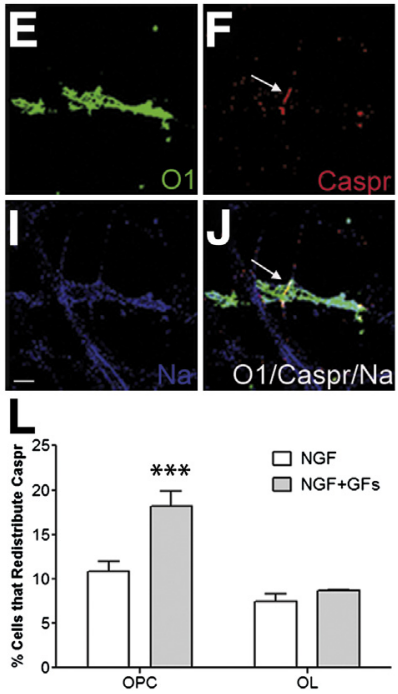

Figure 2 Comparison of $\mathrm{A} 2 \mathrm{~B} 55^{+} \mathrm{OPCs}$ and mature $\mathrm{OLs}$ in co-culture with DRGN. Human adult OPCS (A, C, D, G, and $\mathbf{H})$ or OLs (B, E, F, I, and $\mathbf{J})$ were seeded on established rat DRGNs maintained in DFM with NGF alone or $\mathrm{NGF}^{+}$ GFs (BDNF/IGF-1/PDGF-AA/bFGF) for 5 weeks. Co-cultures were fixed and immunostained either for neurofilaments (NF) (green) and MBP (red) in $\mathbf{A}$ and $\mathbf{B}$, or 01 (green, $\mathbf{C}$ and $\mathbf{E}$ ) and Caspr (red, $\mathbf{D}$ and $\mathbf{F}$ ), or sodium channel (blue, $\mathbf{G}$ and $\mathbf{I}$ ). Merged images are presented in $\mathbf{H}$ and $\mathbf{J}$. In the co-cultures established with OPCs ( $\mathrm{A} 2 \mathrm{~B} 5^{+}$cells), $\mathrm{MBP}^{+}$cells ensheathing multiple DRGN axons are indicated by asterisks in $\mathbf{A}$ and $\mathbf{B}$; fewer ensheathments were observed in co-cultures with the $\mathrm{A}_{2} \mathrm{~B}^{-}$fraction (OLs) in B. Quantitative data are presented in $\mathbf{K}$. Caspr redistribution on axons in presence of ensheathing cells in OPC co-cultures is indicated by arrows in $\mathbf{D}$ and $\mathbf{H}$; nonredistributed Caspr is observed as specks in the background. More limited Caspr (red) redistribution is seen in $\mathrm{OL}$ co-cultures in $\mathbf{F}$ and $\mathbf{J}$. Quantitative data are provided in $\mathbf{L}$. There was no significant redistribution of sodium channels in $\mathbf{G}$ and $\mathbf{I}$. Data represent three independent experiments performed in duplicate for each condition (approximately 200 cells were counted per coverslip). Statistical significance was determined by one-way analysis of variance, followed by Dunnett's test. ${ }^{* *} P<0.001$. Images were acquired by confocal microscopy. Scale bars: $10 \mu \mathrm{m}$.

\section{Comparative Changes in Aapoptosis-Related Gene Expression in OPCs and OLs under Different Culture Conditions}

In initial studies, we compared expression of cell deathrelated genes between A2B5 antibody-selected (OPCs) and nonselected (OLs) cells in an Affymetrix Human Exon gene screen; three separate central nervous system (CNS) donor samples were used. Of genes designated as being cell death relevant [Ingenuity Pathway Analysis (IPA) program version 8.6; Ingenuity Systems, Redwood City, CA] that 

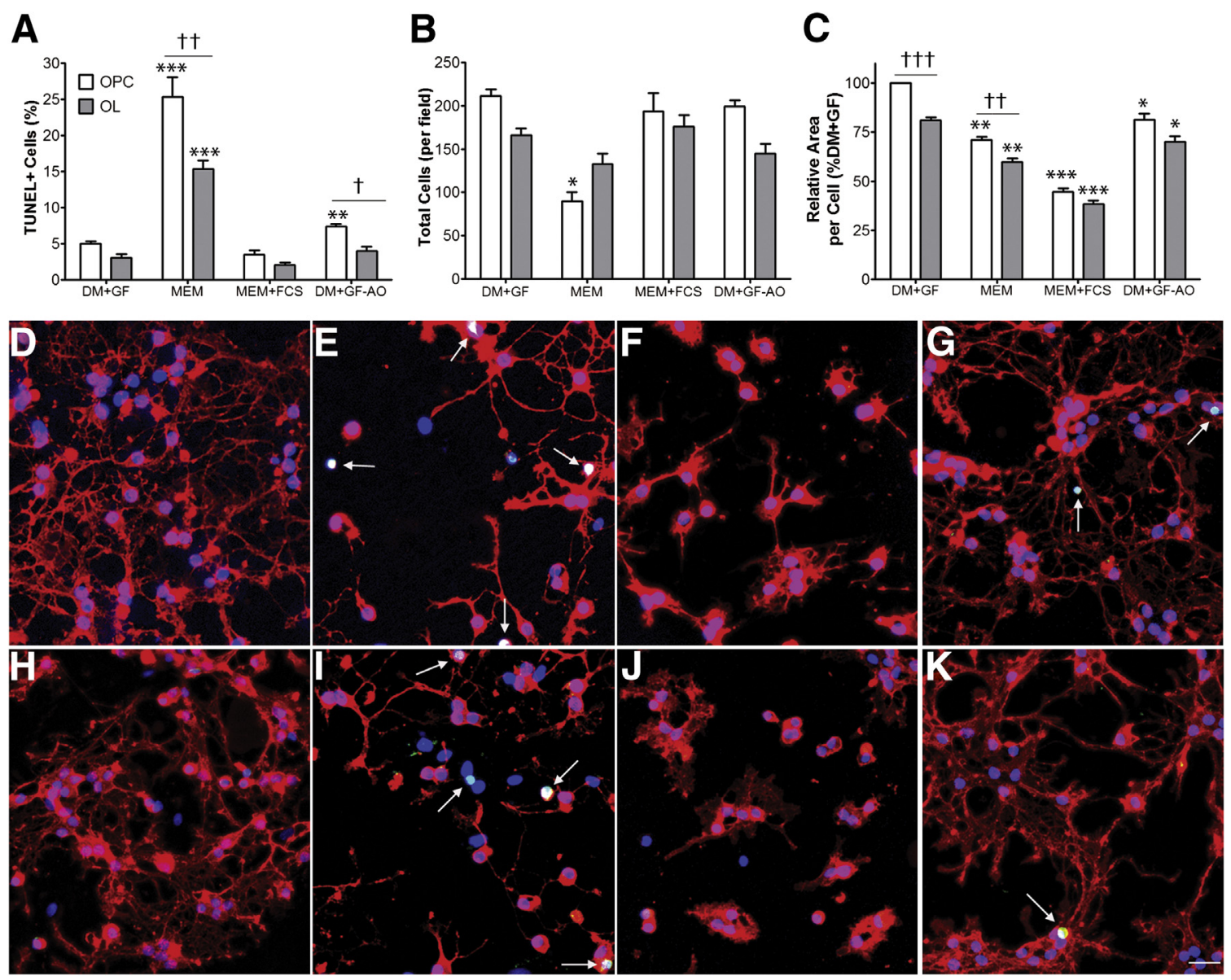

Figure 3 Comparative phenotypic responses of OPCs and OLs under optimal and suboptimal dissociated cell conditions. Percentage TUNEL ${ }^{+}$cells under each condition for either cell type are summarized in $\mathbf{A}$. Total cell numbers per $25 \times$ field are summarized in $\mathbf{B}$. The relative process area per cell calculated as a percentage of that defined in the DM+GF condition for OPCs is shown in C. OPCs ( $\mathbf{D}$ to $\mathbf{G})$ and OLs (H to $\mathbf{K}$ ) cultured in DM+GF (D and $\mathbf{H})$, MEM alone (E and I), MEM+FCS (F and $\mathbf{J})$, or DM+GF-AO (G and $\mathbf{K}$ ) for 6 days before 04 antibody (red) and TUNEL (green), and Hoechst (blue) staining. Arrows in E, G, I, and $\mathbf{K}$ indicate TUNEL ${ }^{+}$cells. Data represent three independent experiments performed in duplicate for each condition. Scale bar $=20 \mu \mathrm{m}$. Statistical significance was determined by two-way analysis of variance, followed by Dunnett's test or Student's $t$-test. ${ }^{*} P<0.05$, ${ }^{* *} P<0.01$, and ${ }^{* * *} P<0.001$ versus corresponding controls; ${ }^{\dagger} P<0.05,{ }^{\dagger \dagger} P<0.01$, and ${ }^{\dagger \dagger} P<0.001$ OPCs versus 0 Ls.

were significantly differentially expressed between the two populations using a paired sample analysis, all were upregulated in the OPC fraction. Supplemental Figure S2 provides a heat map representation of the data.

When the microarray results were fitted to the Kyoto Encyclopedia of Genes and Genomes (KEGG) apoptosis pathway using Partek GS software version 6.6, differentially up-regulated proapoptotic genes were found in the OPC samples (Supplemental Figure S3).

To compare apoptosis pathway-related gene expression from both ex vivo and varying in vitro culture conditions, we used an 84-gene PCR apoptotic pathway array. Data from the 84 apoptosis-related gene PCR array analyses are presented under overall categories of anti- and proapoptotic genes; the latter are further subdivided into intrinsic, extrinsic, and final effecter apoptotic pathway genes (Table 2).

As presented in Figure 4, there was no significant variance in expression of pro- (extrinsic, intrinsic, and effecter) or antiapoptotic genes between the OPC $\left(\mathrm{A} 2 \mathrm{~B} 5^{+}\right)$and $\mathrm{OL}$ $\left(\mathrm{A} 2 \mathrm{~B} 5^{-}\right)$fractions at time of initial cell subset fractionation (day 0). The mean number of antiapoptotic and effecter pathway genes, expressed at $>2$-fold level by OPCs compared to OLs, did not reach statistical significance under any culture condition (Figure 4, A and B; heat map analysis provided in Supplemental Figure S4). Similarly, the intrinsic pathway was not significantly altered (Figure 4C). However, under the most extreme culture condition (MEM alone), there was a net increase ( $>2$ fold) in expression of intrinsic proapoptotic genes for both cell types compared to optimal conditions (DM+GF) (for OPCs, $n=5.3 \pm 1.2$ versus 1.0 $\pm 1.0 ; P<0.05$ ) (Table 3 and Supplemental Figure S5); (for OLs, $n=4.0 \pm 2.1$ versus $0.3 \pm 0.4 ; P=0.11$ ) (Table 3 and Supplemental Figure S6). We did not detect any significant differences under the other culture conditions $(\mathrm{MEM}+\mathrm{FCS}$ or $\mathrm{DM}+\mathrm{GF}-\mathrm{AO}$ versus $\mathrm{DM}+\mathrm{GF})$.

The most significant change in gene expression was observed for the extrinsic proapoptotic gene category that was up-regulated under all culture conditions in the OPC population compared to OLs (approximately 8 to 10 of 23 genes evaluated) (Figure 4D). These included receptors and ligands 


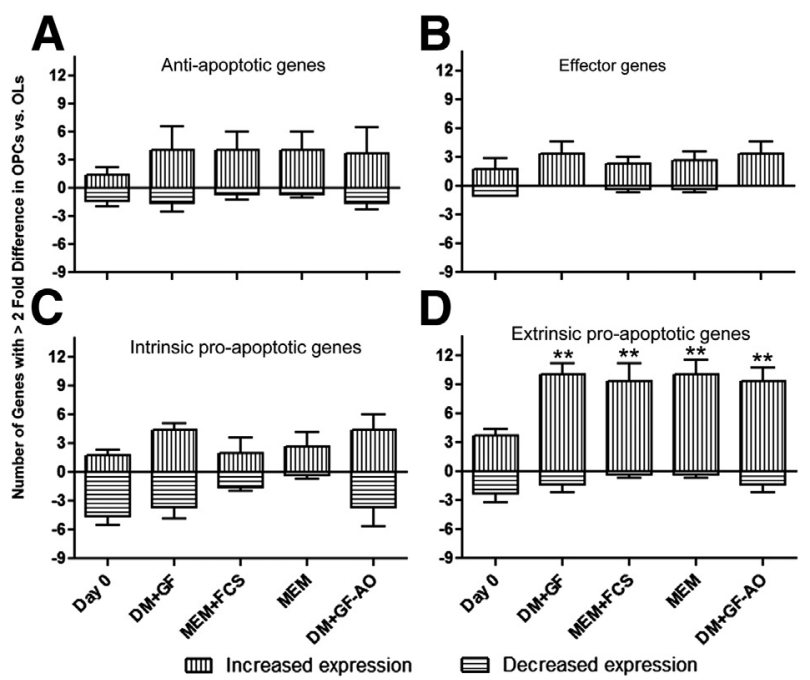

Figure 4 Gene expression changes in OPCs versus oligodendrocytes (OLs). The number of antiapoptotic (A), effector (B), proapoptotic intrinsic (C), or extrinsic (D) genes with increased or decreased expression among the 84 genes included in the PCR apoptosis array between cell types when first isolated (day 0 ) or under different culture conditions at day 6 was calculated by comparing expression between OPCs and OLs in each condition. Increased or decreased expression was based on fold $>2.0(n=3$ samples for each condition). Statistical significance was determined by two-way analysis of variance, followed by Bonferroni's post hoc test. ${ }^{* *} P<$ 0.01 up-regulated versus down-regulated genes.

for cell death-activating molecules, which are members of the TNF receptor (TNF-R) superfamily (Supplemental Figure S4).

To assess whether the increased expression of the extrinsic apoptotic pathway in OPCs could result in enhanced susceptibility to TNF- $\alpha$-mediated injury, we exposed cultures, maintained under optimal (DM+GF) or suboptimal (MEM alone) conditions, to $100 \mathrm{ng} / \mathrm{mL}$ TNF- $\alpha$ for 48 hours (Figure 5). In MEM, both OPC and OLs showed an increase in the percentage of $\mathrm{TUNEL}^{+}$cells after treatment with TNF- $\alpha$. Under both optimal and suboptimal conditions, TNF- $\alpha$ produced a greater increase in the percentage of $\mathrm{TUNEL}^{+}$cells for OPCs compared to OLs $(P<0.05)$.

\section{Discussion}

Our in situ analysis implicates OPCs as a component of the tissue injury that occurs in active demyelinating lesions in MS. The cell markers used to identify OPCs and OLs in white matter are the same as used previously, ${ }^{6}$ having been optimized for use in fixed human tissue sections. The percentage of mature OLs was reduced in all of the actively demyelinating lesions, as well as in the PPWM, compared to control white matter, suggesting that restoration of mature OLs remains insufficient over the time course we have evaluated. OPCs (strongly Olig2 ${ }^{+}$cells) were reduced in 11 of 20 actively demyelinating lesions when compared to non-MS control tissue. In eight of these cases, the relative reduction was greater for OPCs than for mature OLs in the corresponding sample, suggesting that OPCs were more vulnerable to injury mediators in the same microenvironment.
In the other nine cases, OPC numbers were increased in the lesions when compared to non-MS control tissue, suggesting that OPCs have migrated or proliferated to initiate tissue repair. These findings are consistent with experimental studies showing migration or proliferation of OPCs during initiation of remyelination. ${ }^{5,31}$ OPC numbers were also on average increased in PPWM, even in cases where OPC numbers in lesions remained reduced. The low numbers of OPCs in some lesions, despite a marked increase of OPCs in PPWM, may hint also to a disturbed migration of OPCs into MS lesions as described in an earlier study. ${ }^{32}$

Our co-culture studies demonstrate that the cells designated as OPCs (A2B5 selected), rather than the mature OLs, have greater capacity to ensheath axons, the initial step in myelin formation. OPCs selected with the A2B5 antibody from the adult human CNS are able to myelinate following adoptive transfer into the CNS of the dysmyelinated shiverer mouse mutant. ${ }^{33,34}$ Earlier observations showing that mature OLs were present in demyelinated MS lesions led to the postulate that these cells contributed to remyelination. ${ }^{35}$ However, when mature OLs were transplanted into experimentally demyelinated lesions, they failed to remyelinate. ${ }^{36,37}$ Conversely, depletion of OPCs prevented successful remyelination. ${ }^{38}$ The mature OLs used in our study are a post-mitotic cell population that expresses myelin basic protein (MBP) and MOG. ${ }^{26}$ Such cells comprise approximately $30 \%$ of the total numbers recovered from adult human brain specimens, supporting the conclusion that these are previously myelinating OLs and not derived over time in vitro from a relatively small progenitor population. Our microarray analysis further demonstrated that the A2B5-selected cells expressed higher levels of the OPCassociated markers PDGFR $\alpha$ and the protein tyrosine phosphatase, receptor-type, Z1 (PTPRZ1), ${ }^{29}$ further strengthening the notion that these cells are indeed OPCs and that the nonselected fraction consists of mature OLs.

In the OPC-DRGN co-cultures, we observed clustering of Caspr, an indication of axonal response to contact with OPCs. However, the extent of sodium channel redistribution was limited and less than observed previously using fetal human CNS-derived OPCs. ${ }^{39} \mathrm{We}$ cannot determine whether this limited response reflects the experimental paradigm, for example, due to a species difference, or the intrinsic properties of the adult CNS-derived OPCs. We note that rodent OPCs produce extensive myelination of DRGN axons in this system. ${ }^{30}$ Our isolated cell culture observations regarding process outgrowth under optimal conditions by mature OLs indicate that the limited capacity of mature OLs to ensheath axons cannot be attributed to an intrinsic inability to extend cellular processes.

Comparative in Vitro Injury Responses of OPCs and OLs to Metabolic Insults

Our comparative cell survival studies in isolated culture suggest that under all growth conditions, OPCs are more vulnerable to metabolic insults than mature OLs. The 
Table 3 Gene Expression Changes in OPCs or OLs Cultured under Suboptimal Conditions Compared to Optimal Condition

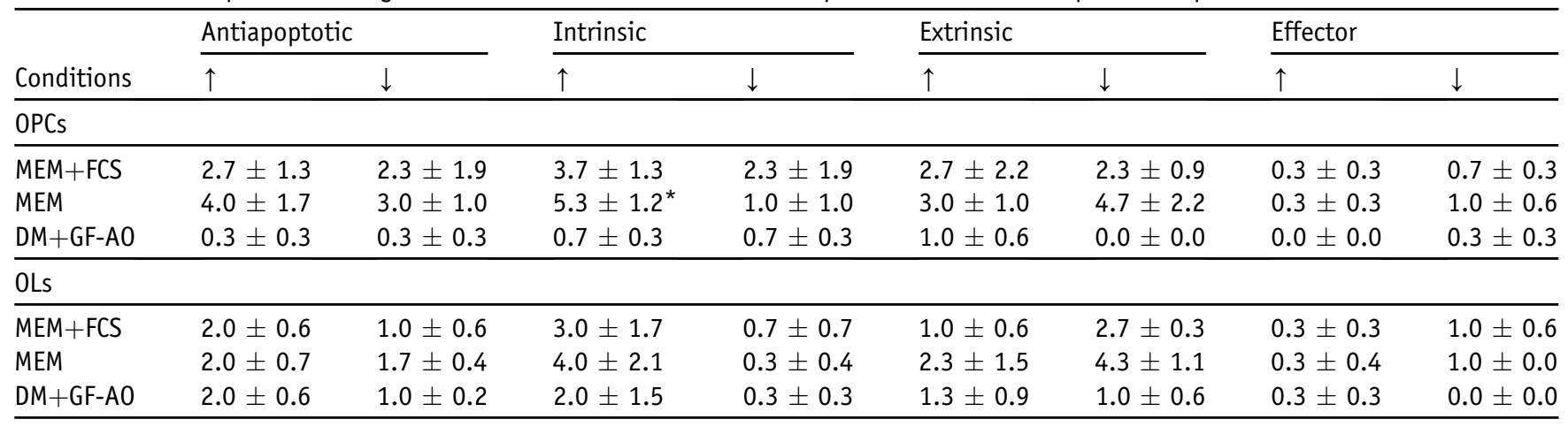

OPCS or OLs maintained in defined culture conditions for 6 days before RNA extraction. Real-time PCR microarray was performed using a human apoptosis $\mathrm{PCR}$ array kit as indicated in methods. The 84 genes were classified into four groups as listed in Table 2. Data are expressed as mean number of genes \pm SEM with $>2$ fold increased $(\uparrow)$ or decreased expression $(\downarrow)$ under suboptimal conditions (MEM+FCS, MEM, DM+GF-A0) compared to optimal condition (DM+GF) in OPCS or OLs. Analyses of all gene changes are presented in Supplemental Figures S3 and S4 as heat maps.

$* P<0.05$.

greatest differences in cell death were observed under the extreme growth deprivation conditions when both growth factors and antioxidants were removed. We note that under such conditions, A2B5 cells selected from the fetal human CNS do not survive at all (data not shown). However, significant differences were also observed under less extreme conditions, such as prolonged culture (3 weeks) in MEM+FCS or when antioxidants alone were withdrawn. With regard to mechanisms of injury, we found a modest increase in the number of intrinsic cell-death pathway genes up-regulated in OPCs versus OLs after 6 days under the most severe culture conditions. We cannot exclude the possibility that intrinsic or final effecter signaling pathway activation would be more apparent if cultures were analyzed at earlier time points or that cell necrosis could also contribute to cell death. Both in vitro and in situ studies demonstrate that ischemia can produce cytotoxic as well as active cell death,

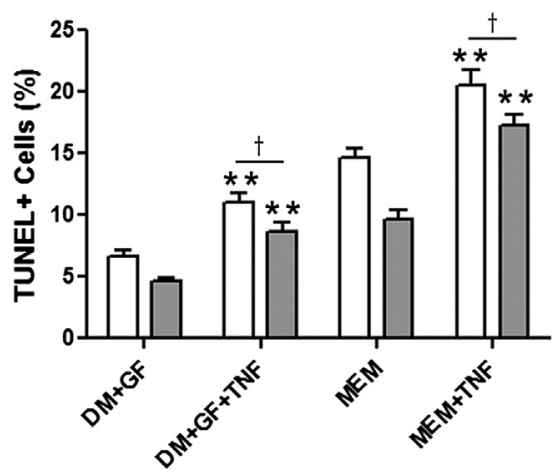

Figure 5 Injury responses of OPCs (white bars) and OLs (grey bars) to TNF $\alpha$ under optimal (DM+GF) and suboptimal (MEM) cell conditions. Cell cultures were exposed to $100 \mathrm{ng} / \mathrm{mL}$ TNF- $\alpha$ for 48 hours under each condition followed by TUNEL assays. Data represent percentage TUNEL ${ }^{+}$ cells in four independent experiments performed in duplicate for each condition. Addition of TNF- $\alpha$ increased percentage TUNEL ${ }^{+}$cells for both $\mathrm{OPCS}$ and $\mathrm{OLs}$ under all conditions. ${ }^{*} P P<0.01$, two-way analysis of variance, followed by Bonferroni's post hoc test. In the presence of TNF- $\alpha$, percentage $\mathrm{TUNEL}^{+}$cells were greater for OPCs than OLs for both the $\mathrm{DM}+\mathrm{GF}$ and MEM conditions. ${ }^{\dagger} P<0.05$, Student's $t$-test. including the death of OLs (reviewed by Dewar et $\mathrm{al}^{40}$ ). Our observations of a maturation-dependent susceptibility of oligodendroglial injury are consistent with earlier studies demonstrating similar results using rodent cells and animal models for periventricular leucomalacia. ${ }^{41,42}$

Under all culture conditions, OPCs up-regulated extrinsic apoptotic pathway genes to an extent greater than OLs. Such responses could be triggered either directly by the selective sensitivity of these cells to the stress of culture conditions compared to OLs or be triggered by molecules of the intrinsic pathway or molecules released by injured or dying cells. Studies on PC12 $2^{43}$ and Jurkat cell lines, ${ }^{44}$ as well as primary dopaminergic neurons, ${ }^{45}$ showed that withdrawal of trophic support, as in our experiments, can induce expression of genes in the extrinsic pathway resulting in cell death. Overexpression of extrinsic pathway signaling receptors and ligands may have specific relevance for continuing injury in MS lesions. ${ }^{46,47}$ We previously showed that inducing sublethal increases in expression of p53, an intrinsic pathway cell death-promoting molecule in OLs by adenovirus transduction resulted in up-regulated expression of members of the TNF-R superfamily, making the OLs more vulnerable to fas- and TNF-related apoptosis-inducing ligand (TRAIL)mediated cell death. ${ }^{48}$ Our experiments further show that the OPCs are more vulnerable to TNF $\alpha$-mediated injury than are the mature OLs when assessed under optimal and suboptimal growth conditions.

\section{Conclusions}

We suggest that vulnerability of human OPCs to conditions that induce injury of mature OLs in MS lesions contribute to the limited remyelination observed in both acute and chronic stages of MS. Studies based on animal models indicate that the severity and duration of an injury is an important determinant of the extent to which remyelination will be limited. Studies using the OL lineage toxin cuprizone indicate that remyelination can occur after repeated 
rounds of injury, but if the injury is sufficiently severe (eg, prolonged exposure to high-dose toxin), recovery will fail to occur. $^{49}$ In MS, repeated or chronic insults to OPCs would be expected. Multiple additional variables are also expected to contribute to failure of remyelination, including the presence of inhibitory molecules, absence of signals needed to recruit and induce OPC differentiation, and compromised integrity of demyelinated axons.

\section{Supplemental Data}

Supplemental material for this article can be found at http://dx.doi.org/10.1016/j.ajpath.2013.04.016.

\section{References}

1. Tripathi RB, Rivers LE, Young KM, Jamen F, Richardson WD: NG2 glia generate new oligodendrocytes but few astrocytes in a murine experimental autoimmune encephalomyelitis model of demyelinating disease. J Neurosci 2010, 30:16383-16390

2. Zawadzka M, Rivers LE, Fancy SP, Zhao C, Tripathi R, Jamen F, Young K, Goncharevich A, Pohl H, Rizzi M, Rowitch DH, Kessaris N, Suter U, Richardson WD, Franklin RJ: CNS-resident glial progenitor/stem cells produce Schwann cells as well as oligodendrocytes during repair of CNS demyelination. Cell Stem Cell 2010, 6:578-590

3. Carroll WM, Jennings AR, Ironside LJ: Identification of the adult resting progenitor cell by autoradiographic tracking of oligodendrocyte precursors in experimental CNS demyelination. Brain 1998, 121(Pt 2): 293-302

4. Franklin RJ, Ffrench-Constant C: Remyelination in the CNS: from biology to therapy. Nat Rev Neurosci 2008, 9:839-855

5. Gensert JM, Goldman JE: Endogenous progenitors remyelinate demyelinated axons in the adult CNS. Neuron 1997, 19:197-203

6. Kuhlmann T, Miron V, Cui Q, Wegner C, Antel J, Bruck W: Differentiation block of oligodendroglial progenitor cells as a cause for remyelination failure in chronic multiple sclerosis. Brain 2008, 131:1749-1758

7. Antel J: New directions in multiple sclerosis therapy: matching therapy with pathogenesis. Can J Neurol Sci 2010, 37(Suppl 2):S42-S48

8. Law M, Saindane AM, Ge Y, Babb JS, Johnson G, Mannon LJ, Herbert J, Grossman RI: Microvascular abnormality in relapsingremitting multiple sclerosis: perfusion MR imaging findings in normal-appearing white matter. Radiology 2004, 231:645-652

9. Lassmann H: Hypoxia-like tissue injury as a component of multiple sclerosis lesions. J Neurol Sci 2003, 206:187-191

10. Fischer MT, Sharma R, Lim JL, Haider L, Frischer JM, Drexhage J, Mahad D, Bradl M, van Horssen J, Lassmann H: NADPH oxidase expression in active multiple sclerosis lesions in relation to oxidative tissue damage and mitochondrial injury. Brain 2012, 135:886-899

11. Lassmann $\mathrm{H}$, van Horssen $\mathrm{J}$ : The molecular basis of neurodegeneration in multiple sclerosis. FEBS Lett 2011, 585:3715-3723

12. Henderson AP, Barnett MH, Parratt JD, Prineas JW: Multiple sclerosis: distribution of inflammatory cells in newly forming lesions. Ann Neurol 2009, 66:739-753

13. Prineas JW, Kwon EE, Cho ES, Sharer LR, Barnett MH, Oleszak EL, Hoffman B, Morgan BP: Immunopathology of secondary-progressive multiple sclerosis. Ann Neurol 2001, 50:646-657

14. Rodriguez M, Scheithauer BW, Forbes G, Kelly PJ: Oligodendrocyte injury is an early event in lesions of multiple sclerosis. Mayo Clin Proc 1993, 68:627-636

15. Mahad D, Ziabreva I, Lassmann H, Turnbull D: Mitochondrial defects in acute multiple sclerosis lesions. Brain 2008, 131(Pt 7):1722-1735

16. Dutta R, McDonough J, Yin X, Peterson J, Chang A, Torres $\mathrm{T}$, Gudz T, Macklin WB, Lewis DA, Fox RJ, Rudick R, Mirnics K,
Trapp BD: Mitochondrial dysfunction as a cause of axonal degeneration in multiple sclerosis patients. Ann Neurol 2006, 59:478-489

17. Zambonin JL, Zhao C, Ohno N, Campbell GR, Engeham S, Ziabreva I, Schwarz N, Lee SE, Frischer JM, Turnbull DM, Trapp BD, Lassmann H, Franklin RJ, Mahad DJ: Increased mitochondrial content in remyelinated axons: implications for multiple sclerosis. Brain 2011, 134:1901-1913

18. Andrews T, Zhang P, Bhat NR: TNFalpha potentiates IFNgammainduced cell death in oligodendrocyte progenitors. J Neurosci Res 1998, 54:574-583

19. Khorchid A, Fragoso G, Shore G, Almazan G: Catecholamine-induced oligodendrocyte cell death in culture is developmentally regulated and involves free radical generation and differential activation of caspase- 3 . Glia 2002, 40:283-299

20. Liu HN, Giasson BI, Mushynski WE, Almazan G: AMPA receptormediated toxicity in oligodendrocyte progenitors involves free radical generation and activation of JNK, calpain and caspase 3. J Neurochem 2002, 82:398-409

21. Fragoso G, Martinez-Bermudez AK, Liu HN, Khorchid A, Chemtob S, Mushynski WE, Almazan G: Developmental differences in HO-induced oligodendrocyte cell death: role of glutathione, mitogen-activated protein kinases and caspase 3. J Neurochem 2004, 90:392-404

22. Ziabreva I, Campbell G, Rist J, Zambonin J, Rorbach J, Wydro MM, Lassmann H, Franklin RJ, Mahad D: Injury and differentiation following inhibition of mitochondrial respiratory chain complex IV in rat oligodendrocytes. Glia 2010, 58:1827-1837

23. Bruck W, Porada P, Poser S, Rieckmann P, Hanefeld F, Kretzschmar HA, Lassmann H: Monocyte/macrophage differentiation in early multiple sclerosis lesions. Ann Neurol 1995, 38:788-796

24. Lucchinetti C, Bruck W, Parisi J, Scheithauer B, Rodriguez M, Lassmann H: Heterogeneity of multiple sclerosis lesions: implications for the pathogenesis of demyelination. Ann Neurol 2000, 47:707-717

25. Ranscht B, Clapshaw PA, Price J, Noble M, Seifert W: Development of oligodendrocytes and Schwann cells studied with a monoclonal antibody against galactocerebroside. Proc Natl Acad Sci U S A 1982, 79:2709-2713

26. Ruffini F, Arbour N, Blain M, Olivier A, Antel JP: Distinctive properties of human adult brain-derived myelin progenitor cells. Am J Pathol 2004, 165:2167-2175

27. Eisenbarth GS, Walsh FS, Nirenberg M: Monoclonal antibody to a plasma membrane antigen of neurons. Proc Natl Acad Sci U S A 1979, 76:4913-4917

28. Sommer I, Schachner M: Monoclonal antibodies (O1 to O4) to oligodendrocyte cell surfaces: an immunocytological study in the central nervous system. Dev Biol 1981, 83:311-327

29. Sim FJ, Lang JK, Waldau B, Roy NS, Schwartz TE, Pilcher WH, Chandross KJ, Natesan S, Merrill JE, Goldman SA: Complementary patterns of gene expression by human oligodendrocyte progenitors and their environment predict determinants of progenitor maintenance and differentiation. Ann Neurol 2006, 59:763-779

30. Fragoso G, Haines JD, Roberston J, Pedraza L, Mushynski WE, Almazan G: p38 mitogen-activated protein kinase is required for central nervous system myelination. Glia 2007, 55:1531-1541

31. Zhang SC, Ge B, Duncan ID: Adult brain retains the potential to generate oligodendroglial progenitors with extensive myelination capacity. Proc Natl Acad Sci U S A 1999, 96:4089-4094

32. Williams A, Piaton G, Aigrot MS, Belhadi A, Theaudin M, Petermann F, Thomas JL, Zalc B, Lubetzki C: Semaphorin 3A and 3F: key players in myelin repair in multiple sclerosis? Brain 2007, 130:2554-2565

33. Windrem MS, Nunes MC, Rashbaum WK, Schwartz TH, Goodman RA, McKhann G 2nd, Roy NS, Goldman SA: Fetal and adult human oligodendrocyte progenitor cell isolates myelinate the congenitally dysmyelinated brain. Nat Med 2004, 10:93-97

34. Windrem MS, Schanz SJ, Guo M, Tian GF, Washco V, Stanwood N, Rasband M, Roy NS, Nedergaard M, Havton LA, Wang S, Goldman SA: Neonatal chimerization with human glial progenitor cells can both remyelinate and rescue the otherwise 
lethally hypomyelinated shiverer mouse. Cell Stem Cell 2008, 2: 553-565

35. Bruck W, Schmied M, Suchanek G, Bruck Y, Breitschopf H, Poser S, Piddlesden S, Lassmann H: Oligodendrocytes in the early course of multiple sclerosis. Ann Neurol 1994, 35:65-73

36. Targett MP, Sussman J, Scolding N, O'Leary MT, Compston DA, Blakemore WF: Failure to achieve remyelination of demyelinated rat axons following transplantation of glial cells obtained from the adult human brain. Neuropathol Appl Neurobiol 1996, 22:199-206

37. Keirstead HS, Blakemore WF: Identification of post-mitotic oligodendrocytes incapable of remyelination within the demyelinated adult spinal cord. J Neuropathol Exp Neurol 1997, 56:1191-1201

38. Blakemore WF, Irvine KA: Endogenous or exogenous oligodendrocytes for remyelination. J Neurol Sci 2008, 265:43-46

39. Cui QL, Fragoso G, Miron VE, Darlington PJ, Mushynski WE, Antel J, Almazan G: Response of human oligodendrocyte progenitors to growth factors and axon signals. J Neuropathol Exp Neurol 2010, 69:930-944

40. Dewar D, Underhill SM, Goldberg MP: Oligodendrocytes and ischemic brain injury. J Cereb Blood Flow Metab 2003, 23:263-274

41. Gerstner B, Buhrer C, Rheinlander C, Polley O, Schuller A, Berns M, Obladen M, Felderhoff-Mueser U: Maturation-dependent oligodendrocyte apoptosis caused by hyperoxia. J Neurosci Res 2006, 84:306-315

42. Gerstner B, DeSilva TM, Genz K, Armstrong A, Brehmer F, Neve RL, Felderhoff-Mueser U, Volpe JJ, Rosenberg PA: Hyperoxia causes maturation-dependent cell death in the developing white matter. J Neurosci 2008, 28:1236-1245

43. Le-Niculescu H, Bonfoco E, Kasuya Y, Claret FX, Green DR, Karin M: Withdrawal of survival factors results in activation of the JNK pathway in neuronal cells leading to Fas ligand induction and cell death. Mol Cell Biol 1999, 19:751-763

44. Caricchio R, D'Adamio L, Cohen PL: Fas, ceramide and serum withdrawal induce apoptosis via a common pathway in a type II Jurkat cell line. Cell Death Differ 2002, 9:574-580

45. Yu LY, Saarma M, Arumae U: Death receptors and caspases but not mitochondria are activated in the GDNF- or BDNF-deprived dopaminergic neurons. J Neurosci 2008, 28:7467-7475

46. Hofman FM, Hinton DR, Johnson K, Merrill JE: Tumor necrosis factor identified in multiple sclerosis brain. J Exp Med 1989, 170: 607-612

47. Dowling P, Shang G, Raval S, Menonna J, Cook S, Husar W: Involvement of the CD95 (APO-1/Fas) receptor/ligand system in multiple sclerosis brain. J Exp Med 1996, 184:1513-1518

48. Wosik K, Antel J, Kuhlmann T, Bruck W, Massie B, Nalbantoglu J: Oligodendrocyte injury in multiple sclerosis: a role for p53. J Neurochem 2003, 85:635-644

49. Ludwin SK: Chronic demyelination inhibits remyelination in the central nervous system. An analysis of contributing factors. Lab Invest 1980, 43:382-387 